\title{
Determinants of the rod and frame effect: The role of retinal size
}

\author{
SHELDON M. EBENHOLTZ \\ University of Wisconsin, Madison 53706
}

\begin{abstract}
In Experiment 1, base-out prisms were used to alter perceived size and distance to a luminous rod and frame while the retinal size remained unchanged. The rod-and-frame effect (RFE) was the same, whether the display was viewed directly or through the prisms. In Experiment 2, one large and one small rod-and-frame display were placed at distances such that they produced identical retinal angles. This was replicated at three different sets of distances. Perceived size and distance of the large and small frame of identical retinal angle interacted with the observation distance, such that at near distances the large frame was perceived as larger and farther than the small frame while, at far distances, both types of estimates converged to a constant value. In contrast, the RFE was identical for the large and small frames matched in retinal angle, but diminished with distance. In both experiments, the RFE varied precisely with variation in retinal angle. Implications of the role of retinal angle in the RFE and for the interpretation of individual differences were discussed.
\end{abstract}

Previous research (Ebenholtz \& Benzschawel, (1977) has established that the magnitude of the rod-and-frame effect (RFE), first investigated by Witkin and Asch (1948), is greatly influenced by the distance between the observer and the luminous frame. Within a range from 1 to $5 \mathrm{~m}$, the RFE approximated a linear inverse function of distance with essentially no RFE at all at $5 \mathrm{~m}$. The question examined in the present paper concerns the mechanisms that conceivably underlie this result.

With increasing distance between the visual display and the subject, three changes occur, each of which could, in principle, cause the reduction in RFE. First, the retinal size of the pattern will vary inversely with distance and, assuming that subjects tend to direct their focus toward the center of the frame, there will result a shift in retinal stimulation from peripheral to foveal. Either one or both of these retinal factors, i.e., the size change or the shift to foveal viewing, could contribute to the reduction in magnitude of the RFE. Second, changes in distance, especially within a few meters, will be accompanied by changes in apparent distance and, third, at distances beyond about $2 \mathrm{~m}$ size constancy, based solely upon the cues of convergence and accommodation, will begin to fail (Leibowitz \& Moore, 1966), with the consequence that detectable changes in apparent size will occur. Thus, changes in retinal size, apparent distance, or apparent size, singly or jointly, may be responsible for the effects of distance on the RFE.

The research was supported in part by Grant MH 13006. The author is grateful to Jonathan Beers for his aid in directing subjects in Experiment 1 and to Judith Callan for directing Experiment 2 and for data analysis.
The following studies explore these possibilities. In Experiment 1, the RFE, obtained when viewing through a pair of base-out prisms, was compared with the RFE determined under normal nonprism viewing. By increasing convergence, the rod and frame was made to appear nearer, and, since retinal size was essentially unaltered, the display appeared smaller as well. If either or both apparent size and apparent distance influence the RFE, this influence should show up as a change in RFE under prism relative to direct-viewing conditions.

\section{EXPERIMENT 1}

\section{Method}

Design. Two groups of eight subjects each viewed a rod-andframe apparatus at a distance of $1.72 \mathrm{~m}$. The task, to set a luminous line to the apparent vertical, was accomplished by all subjects under two conditions. In one, the apparatus was viewed directly, while in the second condition, a pair of $12-\mathrm{D}$ prisms $^{1}$ was placed base out before the eyes. The groups differed only in the prism/ no-prism sequence.

Apparatus. The square frame was made of electroluminescent panels, $106.8 \mathrm{~cm}$ on a side and $2.4 \mathrm{~cm}$ wide. The rod had dimensions of $95.4 \times 2.2 \mathrm{~cm}$, and both rod and frame had a luminance of about $1 \mathrm{~cd} / \mathrm{m}^{2}$. At the viewing distance of $1.72 \mathrm{~m}$, the frame produced a retinal angle of $35.6^{\circ}$, and assuming an interocular axis of $.062 \mathrm{~m}$, a convergence angle $(\gamma)$ of $2.06^{\circ}$. With prisms in place, $\gamma$ increased to $15.81^{\circ}$ corresponding to an optical distance of $22.31 \mathrm{~cm}$.

Subjects were seated in a modified dental chair with the height of the outer canthus raised to the level of the axis around which the frame and line were capable of rotation, the latter at a speed of $1 \mathrm{rpm}$ with the direction controlled by the experimenter. The head was constrained in a chin- and foreheadrest at the upright position.

Procedure. The prism and no-prism conditions were separated by a 7-day interval with the sequence counterbalanced over groups. At each session, with the frame set at $22^{\circ}$ clockwise, the 
subject made four settings of the rod to the apparent gravitational upright, from starting positions alternating at $25^{\circ}$ clockwise and counterclockwise of true vertical. Taped instructions encouraged subjects to rotate the line so that it looked parallel with its gravitational direction, exemplified by the walls of the room, and not to be concerned about the actual orientation of the line. Bracketing of the desired position was permitted until the subject was satisfied. After each session, a black curtain was interposed between the subject and the frame, the subject closed his eyes, and the experimenter recorded the setting from a meter, estimating to $.5^{\circ}$. In Experiment 1, no settings were taken with the frame upright, hence the mean of the four estimates with the tilted frame was taken as the measure of the RFE. At each session, after all line settings, the subjects made estimates in feet and inches of the size and distance to the frame while still in the dark.

Subjects. Thirteen female and three male subjects volunteered to participate. One subject, who, under prism viewing, estimated the frame to be $11 \mathrm{ft}$ on a side at a distance of $35 \mathrm{ft}$ was replaced, because of the extreme score.

\section{Results and Discussion}

Analysis of variance of the line settings for sequence effects (Grant, 1949) yielded no significant effects of order (i.e., 1st task vs. 2nd task) or of prism conditions, but there was a significant difference between groups, $F(1,14)=56.58, p<.01$. However, the difference was not maintained when tested against an error term based upon the sum of squares for subjects within groups (i.e., sequences), $F(1,14)=3.22, p>.05$. Thus, the differences between groups in RFE may be attributed entirely to subject differences. This would seem to be a likely occurrence, given the large individual differences associated with performance on a rod-and-frame task. The means and standard error of each condition are represented in Table 1 . It may be noted that with $\mathrm{df}=7$, each mean differed significantly from zero at $\mathrm{p}<.01$.

Although the prism conditions were without influence on the RFE, both size and distance perception were significantly altered. Analysis of distance estimates, shown in Table 2 , yielded a significant effect of prism conditions, $F(1,14)=18.89$, $\mathrm{p}<.01$, but also a significant sequence effect, beyond that attributable to subject differences between groups, $F(1,14)=5.28, p<.05$. The nature of the effect was such as to cause the second estimate to shift in the direction of the first, and this occurred even with a 1-week interval between sessions. Thus, for example, the prism was more effective in reducing apparent distance when prism viewing was the first condition, and when direct viewing occurred first, distance estimates were relatively larger than when they occurred second. Similar effects of sequence have occurred on the RFE over a 3-week period (Ebenholtz \& Benzschawel, 1977).

Comparisons between prism and no-prism conditions for each sequence separately showed that in each case direct viewing yielded a greater distance estimate than prism viewing with $t(7)=5.65, p<.01$, and $t(7)=2.05, p<.05$, for the prism 1st, no-prism 2nd, and no-prism 1st, prism 2 nd conditions, re- spectively. There is thus little doubt that altered convergence cues effectively reduced the apparent distance to the rod-and-frame display.

Apparent size estimates are shown in Table 3. Analysis of variance showed a significant effect of prisms on apparent frame size, $F(1,14)=27.49$, $\mathrm{p}<.01$, but order was significant, $\mathrm{F}(1,14)=5.82$, $\mathrm{p}<.05$, as well as sequence, $F(1,14)=4.63, p<.05$, the latter reflecting differences in sequence beyond that attributable to subject differences between groups. This pattern of differences between conditions may be interpreted by reference to Table 3 , where direct viewing (i.e., no-prism) produced size estimates close to veridical, i.e., $106.8 \mathrm{~cm}$, whether the estimate was preceded by prism viewing or not. On the other hand, direct viewing did exert a substantial influence on subsequent estimates made with prisms. Comparisons between prism and direct viewing conditions for each sequence separately showed a significant decrease in size estimates under prism viewing only when the prism condition occurred first, $\mathfrak{t}(7)=8.61, \mathrm{p}<.01$.

Overall, the data indicate that where shifts in apparent distance and size were highly significant, these shifts were not accompanied by corresponding changes in the RFE. There is thus no evidence in the present experiment that apparent distance or apparent size contribute to the RFE. On the other

Table 1

Mean RFE and Standard Errors for Prism (Pr) and No-Prism (No-Pr) Conditions of Experiment 1

\begin{tabular}{|c|c|c|c|c|c|c|c|}
\hline \multicolumn{4}{|c|}{ Group 1} & \multicolumn{4}{|c|}{ Group 2} \\
\hline \multicolumn{2}{|c|}{ Pr First } & \multicolumn{2}{|c|}{ No-Pr Second } & \multicolumn{2}{|c|}{ No-Pr First } & \multicolumn{2}{|c|}{ Pr Second } \\
\hline $\mathbf{M}$ & $\sigma_{\mathbf{M}}$ & $\mathbf{M}$ & ${ }^{\sigma} \mathrm{M}$ & $\mathbf{M}$ & $\sigma_{\mathbf{M}}$ & $\mathbf{M}$ & ${ }^{\sigma} \mathbf{M}$ \\
\hline 6.83 & 1.10 & 6.35 & .88 & 3.86 & 1.26 & 3.78 & 1.20 \\
\hline
\end{tabular}

Table 2

Mean Distance Estimates (in Centimeters) and Standard Errors for Prism (Pr) and No-Prism (No-Pr) Conditions of Experiment 1

\begin{tabular}{|c|c|c|c|c|c|c|c|}
\hline \multicolumn{4}{|c|}{ Group 1} & \multicolumn{4}{|c|}{ Group 2} \\
\hline \multicolumn{2}{|c|}{ Pr First } & \multicolumn{2}{|c|}{ No-Pr Second } & \multicolumn{2}{|c|}{ No-Pr First } & \multicolumn{2}{|c|}{ Pr Second } \\
\hline $\mathbf{M}$ & $\sigma_{\mathbf{M}}$ & $\mathbf{M}$ & $\sigma_{\mathrm{M}}$ & $\mathbf{M}$ & $\sigma_{\mathbf{M}}$ & $\mathbf{M}$ & $\sigma_{\mathbf{M}}$ \\
\hline 42.67 & 11.58 & 121.92 & 16.15 & 146.61 & 12.50 & 93.27 & 20.73 \\
\hline
\end{tabular}

Table 3

Mean Size Estimates (in Centimeters) and Standard Errors for Prism (Pr) and No-Prism (No-Pr) Conditions of Experiment 1

\begin{tabular}{|c|c|c|c|c|c|c|c|}
\hline \multicolumn{4}{|c|}{ Group 1} & \multicolumn{4}{|c|}{ Group 2} \\
\hline \multicolumn{2}{|c|}{ Pr First } & \multicolumn{2}{|c|}{ No-Pr Second } & \multicolumn{2}{|c|}{ No-Pr First } & \multicolumn{2}{|c|}{ Pr Second } \\
\hline $\mathbf{M}$ & ${ }^{\sigma} \mathbf{M}$ & $\mathbf{M}$ & $\sigma_{\mathrm{M}}$ & M & $\sigma_{\mathbf{M}}$ & $\mathbf{M}$ & $\sigma_{\mathbf{M}}$ \\
\hline 24.99 & 3.35 & 100.89 & 9.45 & 106.68 & 13.72 & 78.64 & 16.76 \\
\hline
\end{tabular}


hand, it may be recalled that the retinal size of the rod-and-frame display was constant at about $35.6 \mathrm{deg}$ in the prism and direct-viewing conditions. If retinal size is the critical variable, then, since no changes in retinal size occurred, none should be expected in the RFE. The role of retinal size was pursued in Experiment 2, where pairs of frames of different sizes were placed at distances appropriate to produce equal retinal angles. This was done over a range of distances so that, because of size constancy, perceived size and retinal angle were not directly correlated.

\section{EXPERIMENT 2}

\section{Method}

Design. Three groups of 16 subjects each were exposed to a pair of rod-and-frame displays whose linear dimensions were in a 3:1 ratio. Each group was assigned to one of three pairs of distances chosen such that the small and large frame each projected the identical retinal angle. These were, for the small and large frame, respectively, .5 and $1.5 \mathrm{~m}$ for Group $\mathrm{N}$ (near), $1.5 \mathrm{~m}$ and $4.5 \mathrm{~m}$ for Group $M$ (medium distance), and $2.5 \mathrm{~m}$ and $7.5 \mathrm{~m}$ for Group $F$ (far). All subjects set the rod to the apparent vertical in the large and small frame in succession, with the order counterbalanced over subjects within each group. If, on the basis of statistical analyses, data within each group may be pooled over the two frame-size sequences, then the design may be characteriized as a mixed model with frame size as the within-subject factor and with distance varied between groups.

Apparatus. The large frame was described earlier with a slight modification that reduced the luminous area of the rod to $85.2 \times 0.9 \mathrm{~cm}$. The small square frame, also made of electroluminescent panels, was $35.6 \times 0.8 \mathrm{~cm}$. The rod, which rotated around an axis at the center of the frame, was electrically controlled at $1 \mathrm{rpm}$ and had dimensions of $28.4 \times 0.3 \mathrm{~cm}$. Subjects viewed the luminous display from a dental chair with the head maintained upright in a chin-and-temple brace. Eye level was set at the height of the rotation axis of the luminous line, about $1.23 \mathrm{~m}$ above the floor, which was the same for both frames. In the laboratory, the small frame was located along a line approximately $30^{\circ}$ to the right of the large frame, considering the subject as origin. Viewing of the two frames was accomplished without differential head or eye movements by swiveling the subject in the chair so that each frame in turn appeared straight ahead of the subject. Only one frame was visible at a time and an opaque curtain prevented sight of the room and apparatus until the subject was seated and ready to observe the display in the dark. Although, for a given group, the pair of frames produced equal retinal angles, these varied between the three groups. In the near condition, the retinal projection corresponding to a side of one of the frames amounted to $40.8^{\circ}$, while the comparable figures for the medium and far distances were $13.6^{\circ}$ and $8.2^{\circ}$, respectively. Assignment to Groups $N, M$, and $F$ occurred alternately after every two subjects.

Procedure. Subjects made two line settings to the apparent vertical, one from each of two starting positions at $25^{\circ}$ clockwise and counterclockwise of true vertical. This was accomplished first with an upright frame and then repeated with the frame rotated to $22^{\circ}$ clockwise. The procedure was then duplicated with the second frame. The data from the two starting positions for each frame were averaged, and the RFE was measured in terms of the algebraic difference between settings associated with the tilted and upright frames of the same size. ${ }^{2}$ Immediately after the line settings for a given frame size were completed, direct magnitude estimates of the size and distance to the tilted frame were made in feet and inches. In an attempt to reduce variability normally associated with this task, all subjects were shown a foot ruler during the introduction to the experiment, with the comment that size and distance estimates might be required during the study.

Subjects. After replacing three subjects because of problems associated with equipment, a total of 48 volunteers, 40 female and 8 male, took part.

\section{Results and Discussion}

RFE. The RFE was analyzed separately at each distance to determine whether the sequence in which subjects received the two frame sizes differentially affected the line settings. At the near and medium distances, analysis of variance (Grant, 1949) showed a significant effect of sequence, $F(1,14)=127.16$ and 36.24 , respectively, with $\mathrm{p}<.01$ in each case. But the sequence effect disappeared when tested against the error term based upon differences between subjects within sequence. The significant effect originally obtained can therefore be interpreted as reflecting the large individual differences typically obtained in measures of the RFE. At the far distance, there was no significant sequence effect at all, due to individual differences or otherwise. This outcome is consistent with previous research showing the RFE to vary inversely with distance (Ebenholtz \& Benzschawel, 1977), since individual differences must of necessity diminish as the RFE converges toward zero. The two remaining factors tested in the analysis were order (i.e., first task vs. second task) and frame size, and at none of the three distances was either factor significant at $p<.05$. In the absence of genuine sequence effects, the data were pooled over sequence at each distance and a mixedmodel analysis of variance was undertaken with frame size as the within-subject factor and distance as between-subjects factor. A plot of these data is represented in Figure 1, where the RFE is shown as a function of retinal angle of the frame. As the function suggests, distance was significant, $F(2,45)=$ $6.86, p<.01$, thereby directly corroborating previous research showing the RFE to diminish with distance. That retinal angle rather than distance as such is the critical factor in the RFE is suggested by the finding that neither frame size nor the interaction of frame size and distance was significant. Since the frames at each distance were matched in retinal angle, no effect of frame size should be expected on the retinal size hypothesis.

Apparent size. Estimates of perceived size were analyzed for sequence effects and significant values were obtained at all three distances, attributable to individual differences as such. At near, medium, and far distances, analyses yielded $F(1,14)=30.87$, 19.26 , and 10.01 , respectively, with $p<.01$ in each case. Furthermore, at the near distance only, a sequence effect beyond that attributable to individual differences proved to be significant, $F(1,14)=8.42$, $p<.02$. For this reason, subsequent analyses were 


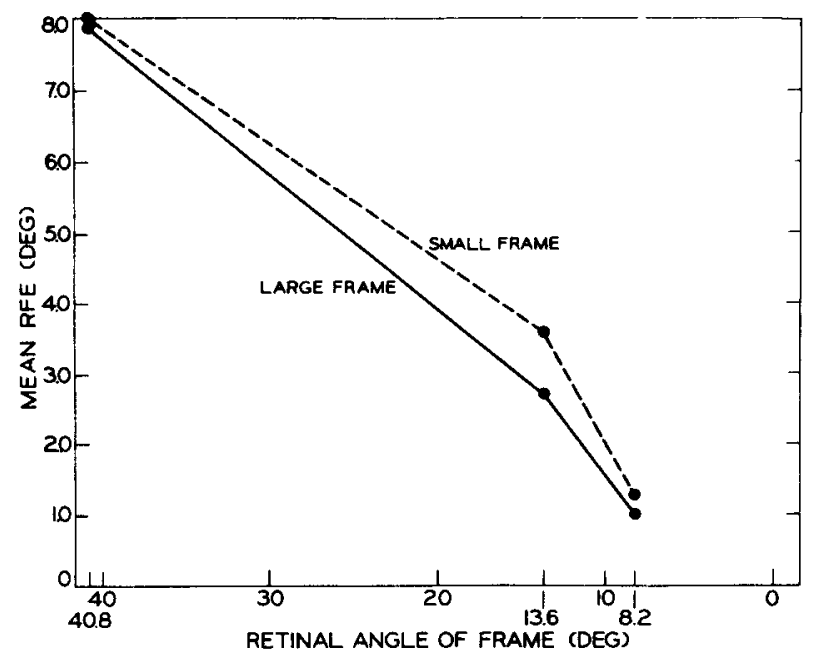

Figure 1. Mean rod-and-frame effect as a function of the retinal angle of one side of each frame.

performed on the size estimates of each sequence separately.

In Figure 2, mean size estimates are shown by sequence as a function of the projected angle of the frame at each distance. The nature of the sequence effect which, as expected, is apparent primarily at the near distance, is such that the frame-size estimate taken second was influenced in the direction of the size of the frame seen first. Thus, in sequence $L \rightarrow S$, the small frame was overestimated, while in sequence $\mathrm{S} \rightarrow \mathrm{L}$, the large frame was underestimated. There was, in addition, an overestimation of the large frame when it was presented first, and this may be due in part to an overestimation in distance. In fact, it is possible that the obtained sequence effects on size actually were mediated by inappropriate distance perception, and this will be taken up below.

Analysis of variance of the size estimates taken during the $\mathrm{L} \rightarrow \mathrm{S}$ sequence yielded significant effects of distance, $F(2,21)=9.94, p<.01$, frame size, $F(1,21)=23.54, p<.01$, and the interaction of the two, $F(2,21)=15.39, p<.01$. The same pattern was exhibited in sequence $S \rightarrow L$, with significant effects of distance, $F(2,21)=3.88, p<.05$, frame size, $F(1,21)=29.47, p<.01$, and their interaction, $F(2,21)=10.08, p<.01$. The fact that estimates of the small frame changed less rapidly with retinal angle, i.e., they have lower slope, than those associated with the large frame may be understood as resulting from a greater degree of size constancy for the former over the latter. The small frame was viewed over a range of .5 to $2.5 \mathrm{~m}$ with only the cues of accommodation and convergence, and, as might be anticipated from the work of Leibowitz and Moore (1966), size constancy should be high at these distances. For the small frame, it was virtually perfect when estimates uninfluenced by prior settings are considered (viz., the small frame in sequence $\mathrm{S} \rightarrow \mathrm{L}$ ). The large frame was seen over a range of 1.5 to $7.5 \mathrm{~m}$, and, since the distance over which convergence and accommodation could be effective was rapidly exceeded, size constancy rapidly fell off.

Could the changes in perceived size have influenced the RFE as represented in Figure 1? While both the RFE and perceived size diminished with distance, ${ }^{3}$ large and significant differences in perceived size occurred between the large and small frames, and these were totally without corresponding changes in the magnitude of the RFE. Apparent size thus cannot serve as an account of the equivalence in RFE for both large and small frames having identical retinal angles.

Apparent distance. Estimates of apparent distance were analyzed separately at each location (i.e., near, medium, and far) for possible sequence effects. At the near position, a genuine sequence effect, beyond that due to individual differences, was obtained, $F(1,14)=10.74, p<.01$. For this reason, subsequent analyses were performed on the data of each sequence separately. These distance estimates are represented in Figure 3 as a function of the distance to each frame. In order to compare directly the distance estimates to the small and large frame of identical visual angle, the abscissa was transformed in terms of the inverse of the retinal angle (i.e., yielding a scale proportional to the actual changes in distance between near, medium, and far positions. The most compelling aspect of the functions represented in Figure 3 is the difference in apparent distance to the two frames at the near range and the convergence of distance estimates with increasing distance. These observations were confirmed by statistical analyses which yielded, for sequence $\mathrm{L} \rightarrow \mathrm{S}$,

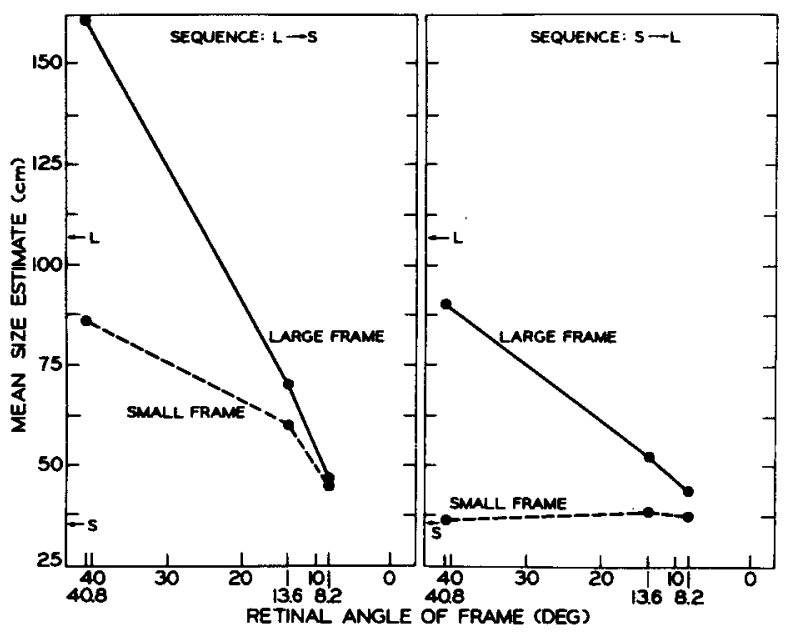

Figure 2. Mean size estimates of one side of each frame as a function of its retinal angle, separately for each sequence. Arrows on ordinate marked $L$ and $S$ indicate actual size of large and small frame, respectively. 


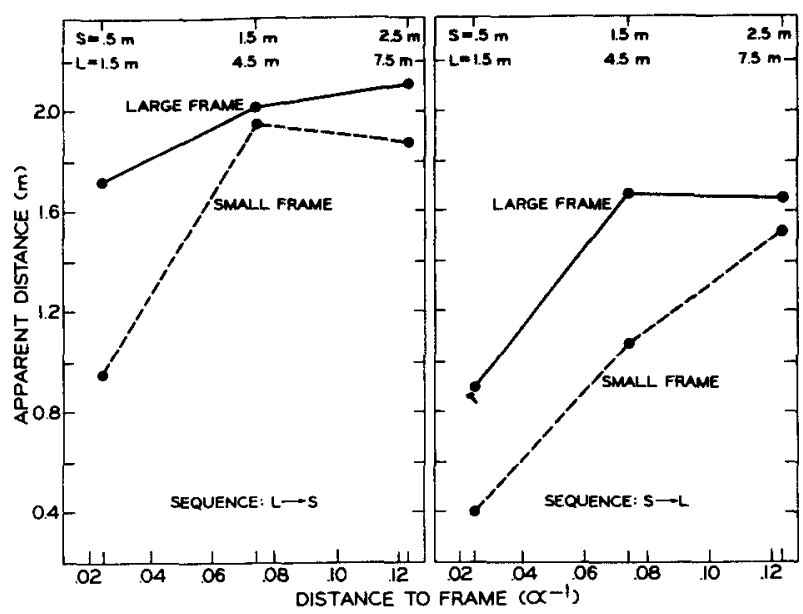

Figure 3. Apparent distance to small (S) and large (L) frame, separately for each sequence, as a function of distance. Abscissa is scaled in terms of the inverse of the retinal angle $\left(\alpha^{-1}\right)$ of the frame. Actual distances to each frame are represented at the top of the figure.

a significant effect of frame size, $F(1,21)=32.59$, $\mathrm{p}<.01$, and a significant interaction of frame size with distance, $F(2,21)=11.72, p<.01$. For sequences $S \rightarrow L$ the main effect of distance was significant, $F(2,21)=9.14, p<.01$, as was frame size, $F(1,21)=28.70, p<.01$. The interaction of the two was significant as well, $F(2,21)=3.47$, $\mathrm{p}=.05$.

If changes in apparent distance were to serve as the basis for the RFE, then one should expect to find an interaction of frame size with distance in the pattern of RFE data. But, in fact, as Figure 1 demonstrates, this was not the case. Instead, the identical RFE was produced by the large and small frame when the retinal angles at each distance were equal, and this outcome cannot be deduced from the relation between the large and small frame conditions expressed in the apparent distance function.

It may be noted in passing that the sequence effects exhibited by the distance estimates were isomorphic with those found in the analysis of the size data. Thus, in sequence $\mathrm{L} \rightarrow \mathrm{S}$, for reasons that are not evident, the distance to the large frame at the near position was overestimated, and this tendency carried over to the small frame as well. In contrast, sequences $S \rightarrow L$ produced underestimation of the distance to the small frame, and this then carried over to the estimates of distance to the large frame. The fact that, at each position (i.e., near, medium, or far), the two frames produced equal retinal angles may have contributed to the sequence effects insofar as there was no difference in the relative retinal angles that otherwise might have led to differences in perceived distance. It is of further interest to note that, at the far distances, subjects' estimates of the distance converged to a value around $2 \mathrm{~m}$. Since only the oculomotor cues of convergence and accommodation could mediate distance perception in the dark, and since the large frame at $7.5 \mathrm{~m}$ exceeded the distance over which these cues are effective, the limiting distance of $2 \mathrm{~m}$ is in need of explanation. This result could reflect the operation of the "specific distance tendency" (Gogel, 1972), i.e., a tendency to perceive objects at some fixed distance when the cues are relatively impoverished. Alternatively, it is conceivable that, with objects at far distances, the oculomotor system may signal not actual distance as such, but that the object in question is at a distance not less than $2 \mathrm{~m}$. In other words, the absence of convergence and accommodative cues may itself signal some minimum observation distance. This possibility is consonant with the approach of Owens and Leibowitz (1976) in advancing the premise that the convergence cue mediates distance perception even under impoverished cue conditions. ${ }^{4}$

\section{GENERAL DISCUSSION}

In both experiments, systematic changes in apparent size and distance to the frames were unaccompanied by corresponding changes in the RFE. On the other hand, when retinal size was kept constant as in Experiment 1, and at the near, medium, and far positions, respectively, in Experiment 2, the RFE also was constant, whereas changes in retinal size always produced corresponding changes in the RFE. The conclusion to be drawn is that retinal size controls the RFE. This has several implications, one of which relates to the proposal of Gogel and Newton (1975) that the RFE should increase with increasing convergence distance to the rod, given that the relative depth between rod and frame and frame-tilt magnitude were held constant. The results of Experiment 1 , shown in Table 1 , given no indication of such an outcome even though large variations in both convergence and apparent distance had occurred. Likewise, the results of Experiment 2, Figure 1, also fail to confirm this possibility since frames of equal retinal size but at different distances yielded equivalent RFEs. It is conceivable that the different methods of measuring the RFE in the present study and that of Gogel and Newton (1975) may somehow contribute to the disparate results, but in any event, without further confirmation, the proposed role of distance in the RFE is dubious.

Second, large and small frames when viewed at the same distance should produce different magnitudes of the RFE. This is evident in Figure 1, in the comparison of the mean RFE of the large frame $\left(7.9^{\circ}\right)$ at the near position and of the small frame $\left(3.6^{\circ}\right)$ at its medium location where both frames were at $1.5 \mathrm{~m}$. It also appears to be the case that any frame 
that produces a retinal angle of about $10^{\circ}$ or less will yield a small RFE, probably under $2^{\circ}$. Accordingly, the low magnitude effects of, e.g., Beh, Wenderoth, and Purcell (1971), Ong and Kessinger (1971), and Wenderoth (1974), of about $1.5^{\circ}$ and under can be explained by their use of frames of the order of $4.5^{\circ}$ to $8^{\circ}$ of retinal angle. Indeed, when such small retinal angles are employed, there is a question of whether a phenomenon different from the high magnitude RFE is involved. That different effects emerge is shown by the study of the apparent vertical as a function of the angular orientation of the frame. Beh, Wenderoth, and Purcell (1971) worked with a frame that projected $7.6^{\circ}$ (viz., 8 in. at $5 \mathrm{ft}$ ) and found a nonmonotonic function with settings in the direction of the frame at $15^{\circ}$, opposite frame tilt at $30^{\circ}$, and a similar pattern for frame tilts from $45^{\circ}$ to $90^{\circ}$, although, as noted above, no effect exceeded $1.5^{\circ}$. In contrast, Graf (1966) worked with a frame that projected a visual angle of about $42.0^{\circ}$ and obtained mean RFEs that ranged from $0^{\circ}$ to $8^{\circ}$. These approximated a negatively increasing monotonic function of frame tilt from $0^{\circ}$ to $30^{\circ}$ with a slight drop at $45^{\circ}$.

Further support for the contention that frames of large retinal angle do not produce negative RFEs derives from the original study of Witkin and Asch (1948), in which the projected frame angle was about $38.2^{\circ}$. With respect to their $15^{\circ}$ and $30^{\circ}$ frame-tilt conditions, Witkin and Asch (1948) noted that "errors were always, except for a single $\mathrm{S}$, in the direction of the tilt of the frame" (p. 774). For the $28^{\circ}$ frame tilt, "the rod was tilted to the opposite side of the true $\mathrm{V}$ and $\mathrm{H}$ from the frame in 18.1 percent of the judgments" (Witkin \& Asch, 1948, p. 766). It thus appears doubtful that, had the signed deviations been used, any of the mean settings for frame tilts of $15^{\circ}, 28^{\circ}$, and $30^{\circ}$ would have been negative. ${ }^{5}$

Wenderoth (1974) has argued that the differences between the rod-and-frame test (RFT) of Witkin and Asch (1948) and the rod-and-frame illusion (RFI) of Wenderoth and his colleagues stem largely from the method used by Witkin and Asch (1948) to calculate the error in rod setting. Their method was to ignore the direction of error, averaging the absolute deviations from vertical (or horizontal). I agree with Wenderoth that this technique is logically inconsistent with the definition of the RFE as an error in rod placement in the direction of frame tilt. ${ }^{6}$ How else could the "field-dependent" category be understood? But, in light of the present results and those of Graf (1966), based upon the algebraic differences between signed errors, it is unlikely that the studies of Beh et al. (1971) and Wenderoth (1974) can be reconciled with the results of Witkin and Asch (1948) merely in these terms. Rather, the projected size of the rod and frame would seem to be the critical variable, especially in accounting for the different error functions with frame tilt and the altered overall magnitude of effect. It follows that small- and largeframe phenomena should not be identified with each other.

A third implication concerns the finding that a tilted room yields greater effects than a luminous frame (Witkin \& Asch, 1948). This was attributed to the possibility that "the more highly structured the surrounding visual framework the greater its influence on perception of the upright" (Witkin \& Asch, 1948 , p. 780). A recent study, however, (Singer, Purcell, \& Austin, 1970) found that the addition of structure, in the form of stripes or furniture, to a tilted room had effects only at $45^{\circ}$ of room tilt, a position where structure could be used to "disambiguate" the direction of room tilt and thereby increase the mean signed error score. From the present results, the hypothesis to account for the difference between frame and room is straightforward, i.e., the differences occur because of the greater retinal size of the room relative to the frame. Thus, all other factors held constant, ${ }^{7}$ a relatively small room should yield a RFE that is lower in magnitude than that of a correspondingly large frame, regardless of room structure.

It is of further interest, in this context, to note that a relatively large circular field of tilted lines, with retinal angle of about $53.13^{\circ}$, produced only a $2.22^{\circ}$ deviation in the direction of the field which was tilted at $22.5^{\circ}$ (Singer et al., 1970). It would thus appear that important differences exist between gratings and other frames of reference, especially with respect to the role of retinal angle.

A fourth implication of the present study stems from consideration of the question of why the RFE diminishes with increasing observation distance, as shown by Ebenholtz and Benzschawel (1977). The immediate answer is that the RFE is dependent upon retinal size which, in turn, is modulated by changes in distance. But why is retinal size critical? A compelling interpretation of the effects of retinal size is possible in light of recent work on induced motion of the self (e.g., Brandt, Dichgans, \& Koenig, 1973; Dichgans, Held, Young, \& Brandt, 1972; Held, Dichgans, \& Bauer, 1975). These studies indicate a profound effect of peripheral, as opposed to foveal, retinal stimulation on both induced selfmotion and on apparent body tilt when stimulus motion is around the line of sight. Since change in retinal size of a fixated frame with distance is confounded with a shift in image location from periphery to fovea, it is plausible to assume that analogous mechanisms underlie the control of spatial orientation in the case of moving as well as static displays. The rod-and-frame effect may, on this account, be regarded as a static version of a class 
of induction effects based upon moving patterns of stimulation.

Consistent with the identification of moving and static displays is the finding that the tilted luminous frame is capable of influencing both apparent object orientation as well as the apparent orientation of the self (Ebenholtz \& Benzschawel, 1977), just as dynamic stimulation yields joint effects on apparent object and self-orientation (Dichgans et al., 1972). Both sets of effects seem to reflect a fundamental division of function associated with the visual projection system (e.g., Schneider, 1967; Trevarthen, 1968) into areas related to search, fixation, identification, and perhaps grasping, associated, on the one hand, with the fovea and, on the other, with the control of spatial orientation of the self, and object localization associated with projection from the peripheral retina.

A final implication to be drawn bears on the significance of individual differences in the RFE. Witkin and his colleagues have argued that such individual differences are rather pervasive in the life of the individual, reflecting his "cognitive style" (Witkin, Goodenough, \& Karp, 1967; Witkin \& Oltman, 1967). The present results do not bear directly on this issue, but they do suggest a physiological basis for individual differences in the RFE. According to the interpretation offered above, subjects who show large errors in the direction of the frame, viz., field-dependent subjects, reflect the operation of the peripheral organization devoted to the control of spatial orientation, while fieldindependent subjects reflect the foveal organization presumably subserving the function of target identification, etc. (Johnson, Leibowitz, Millodot, \& Lamont, 1976; Trevarthen, 1968). Since, with relatively small visual angles of the frame, all subjects become field independent, the defining characteristic separating individuals may simply be the extent of development of the peripheral system. Accordingly, extreme field-independent behavior, especially with frames projecting large retinal angles, may actually represent an anomalous state associated with a deficit in the peripheral orientation control system. ${ }^{8}$

\section{REFERENCES}

Beh, H. C., Wenderoth, P. M., \& Purcell, A. T. The angular function of a rod-and-frame illusion. Perception \& Psychophysics, 1971, 9, 353-355.

Brandt. Th., Dichgans, J., \& Koenig, E. Differential effects of central versus peripheral vision on egocentric and exocentric motion perception. Experimental Brain Research, 1973, 16. 476-491.

Dichgans, J., Held, R., Young, L. R., \& Brandt, T. Moving visual scenes influence the apparent direction of gravity. Science, 1972, 178, 1217-1219.

Ebenholtz, S. M., \& Benzschawel, T. L. The rod and frame effect and induced head tilt as a function of observation distance. Perception \& Psychophysics, 1977, 22, 491-496.
GoGEL, W. C. Scalar perceptions with binocular cues of distance. American Journal of Psychology, 1972, 85, 477-497.

Gogel, W. C., \& Newton, R. E. Depth adjacency and the rodand-frame illusion. Perception \& Psychophysics, 1975, 18, 163-171.

GRAF, R. G. The relationship between perceived head position and the perception of the vertical. Unpublished MA Thesis, Department of Psychology, Connecticut College, 1966.

GRANT, D. W. The statistical analysis of a frequent experimental design. American Joumal of Psychology, 1949, 62, 119-122.

Held, R., Dichgans, J., \& Bauer, J. Characteristics of moving visual scenes influencing spatial orientation. Vision Research, $1975,15,357-365$.

Johnson, C. A., Leibowitz, H. W., Millodot, M., \& Lamont, A. Peripheral visual acuity and refractive error: Evidence for "two visual systems"? Perception \& Psychophysics, 1976, 20, $460-462$.

LEIBOWITZ, H., \& MOORE, D. Role of changes in accommodation and convergence in the perception of size. Joumal of the Optical Society of America, 1966, 56, 1120-1134.

ONG, J., \& Kessinger, D. J. Perception of verticality with a rod and frame apparatus. American Journal of Optometry, Archives of the American Academy of Optometry, 1971, 48, 662-666.

OWens, D. A., \& Leibowitz, H. W. Oculomotor adjustments in darkness and the specific distance tendency. Perception \& Psychophysics, 1976, 20, 2-9.

Schneider, G. E. Two visual systems. Science, 1967, 163, $895-902$.

Singer, G., Purcell, A. T., \& Austin, M. The effect of structure and degree of tilt on the tilted room illusion. Perception \& Psychophysics, 1970, 7, 250-252.

TREVARTHEN, C. B. Two mechanisms of vision in primates. Psychologische Forschung, 1968, 31, 299-337.

WENDERoth, P. M. The distinction between the rod-and-frame illusion and the rod-and-frame test. Perception, 1974, 3 , 205-212.

Witkin, H. A. Perception of body position and of the position of the visual field. Psychological Monographs, 1949, 63, Whole No. $302,1-46$.

WITKIn, H. A., \& Asch, S. E. Studies in space orientation. IV. Further experiments on perception of the upright with displaced visual fields. Journal of Experimental Psychology, 1948, 38, $762-782$.

Witkin, H. A., Goodenough, D. R., \& KARP, S. A. Stability of cognitive style from childhood to young aduithood. Journal of Personality and Social Psychology, 1967, 7, 291-300.

Witkın, H. A., \& Oltman, P. K. Cognitive style. International Journal of Neurology, 1967, 6, 119-137.

\section{NOTES}

1. In pilot studies, the RFE was measured in two groups of 16 subjects each, while viewing through 4-D prisms, placed either base out or base in. Both groups exhibited a significant RFE but did not differ as a function of the direction of prism base. The present study enhanced the convergence effects by increasing prism power and, in so doing, caused a noticeable stereo distortion because of the gradient of optical displacement along the prism base-apex axis and the transformation of vertical to curved images. To the subject, the frame appeared to be concave.

2. A reviewer has suggested that an upright frame as a control condition actually might increase the incidence of settings in alignment with the frame in the tilted-frame condition. There is no direct evidence on this point, but it is clear that subjects did not produce an RFE of the same magnitude as the frame tilt. In any event this possibility does not differentially affect the various experimental conditions. See Ebenholtz and Benzschawel (1977) for additional comments on this control procedure.

3. Even this limited correspondence is contradicted by the high level of size constancy for the small frame in the $S \rightarrow L$ sequence. 
4. Owens and Leibowitz (1976) argue that, under impoverished cue conditions, the convergence assumes an intermediate value, corresponding to the level of convergence in the dark, that signals distance by underestimating the distance to far objects and overestimating the distance to near ones.

5. Witkin and Asch (1948) also reported that "some of the errors were in the opposite direction from the frame tilt" (p. 774) when a frame was rotated $60^{\circ}$ counterclockwise. They pointed out, however, that such a tilt angle could also be seen as $30^{\circ}$ clockwise. Consequently, even the data of the $60^{\circ}$ frame tilt are not inconsistent with the hypothesis that negative RFEs are not associated with frames of large retinal angle.

6. As already noted, some of their subjects gave negative effects, i.e., settings opposite frame tilt, and Witkin and Asch (1948) provided several observations to support the possibility that these subjects misplaced the apparent top of the frame. If so, then even negative settings could be construed as errors in the direction of frame tilt, providing the latter was individually defined for each subject. Although not stated as such by Witkin and Asch (1948), this could serve as the rationale for the use of unsigned errors.
7. One of the factors typically not held constant in the two conditions is the visibility of the self. Sight of the body does not occur with the luminous frame, but is quite likely where the subject is seated within the tilted room (Witkin, 1949). The potential conflict between the visual representation of the tilted room and visible body position may be a contributing factor to the RFE (Ebenholtz \& Benzschawel, 1977).

8. Early development of the peripheral orientational system should serve as an aid to the development of early walking in infants, since proper postural orientation would be signaled by upright frames of reference in everyday environments. Conversely, walking would be retarded in infants who were largely field independent. Sex differences showing greater RFEs in females may reflect these differences in early development of the orientational control system.

(Received for publication May 16, 1977; revision accepted September 12, 1977.) 